\title{
st \\ O projeto de psicologia científica de Edward Tolman
}

\author{
Carlos Eduardo Lopes
}

\begin{abstract}
茴
RESUMO

Os projetos de psicologia científica enfrentam pelo menos duas ameaças. A primeira delas surge quando uma proposta de psicologia tenta seguir os cânones da ciência moderna. Nesse caso, torna-se necessário “objetivar o fenômeno psicológico", o que, geralmente, é feito por meio da sua tradução em termos fisiológicos. Mas, nesse ponto, a especificidade da psicologia é ameaçada pelo reducionismo fisiológico. A segunda ameaça aparece quando um projeto de psicologia tenta evitar o reducionismo fisiológico defendendo a natureza subjetiva irredutível do fenômeno psicológico. No entanto, isso coloca uma série de dificuldades metodológicas. Dessa maneira, a segunda ameaça às propostas de psicologia científica é o mentalismo, que impõe limitações ao emprego do método científico. O presente ensaio tem o objetivo de mostrar como o projeto de psicologia científica de Tolman pode ser visto como uma alternativa a essas duas ameaças. Em primeiro lugar, examinamos em que medida a concepção de comportamento como fenômeno molar afasta Tolman do reducionismo fisiológico. Em seguida, analisamos algumas dificuldades decorrentes dessa proposta, em especial, as limitações inerentes à epistemologia realista - adotada inicialmente por Tolman. Por fim, apresentamos a proposta tolmaniana de superação dessas dificuldades: trata-se da analogia entre teorias científicas e mapas, que revela a adoção de uma epistemologia instrumentalista por Tolman.
\end{abstract}

Palavras- Ghave • Tolman. Comportamento. Mapas. Reducionismo fisiológico. Mentalismo.

\section{INTRODUÇÃO}

Qualquer projeto de psicologia científica tem que enfrentar pelo menos duas ameaças. A primeira delas surge quando esse projeto tenta seguir à risca os cânones do cientificismo. Como a ciência moderna fundamenta-se na dicotomia objetivo-subjetivo com uma evidente predileção pelo primeiro termo -, uma de suas características é a exclusão do sujeito psicológico, entendido como um conjunto de vieses subjetivos, na produção do conhecimento científico (cf. Figueiredo, 2003). Com isso, uma psicologia científica vê-se obrigada a negar a natureza mental do fenômeno que pretende estudar. Em outras palavras, para que a psicologia possa ser considerada uma ciência torna-se necessário "objetivar” os fenômenos psicológicos. Uma das maneiras mais 
evidentes de se fazer isso é defender a natureza fisiológica desses fenômenos; o que, aliás, pode ser facilmente encontrado em certos projetos contemporâneos das chamadas neurociências (cf. Araujo, 2003).

O problema é que, seguindo essa estratégia de substituição do mental pelo fisiológico, a psicologia perde sua especificidade. Em outras palavras, nesse ponto a psicologia científica já não se distingue mais da fisiologia. A primeira ameaça aos projetos de psicologia científica é, portanto, o reducionismo fisiológico, que tem como resultado a conversão da psicologia em fisiologia.

A segunda ameaça a um projeto de psicologia científica surge quando se tenta evitar o objetivismo, e o consequente reducionismo, defendendo-se a natureza subjetiva e irredutível do fenômeno psicológico. No entanto, ao fazer isso, surge uma série de dificuldades metodológicas no estudo do fenômeno psicológico. Afinal, como é possível estudar cientificamente algo que resiste aos métodos de observação típicos da ciência? Essa dificuldade, bem como as críticas imanentes a ela, tiveram que ser enfrentadas por muitas propostas de psicologia científica que adotaram o método introspectivo. Dessa forma, a segunda ameaça à psicologia científica é o mentalismo, ${ }^{\mathbf{1}}$ que encontra dificuldades metodológicas para alcançar o ideal científico.

Dessa forma, os projetos de psicologia científica parecem enfrentar o seguinte dilema: quando se aproximam da ciência, perdem seu objeto, bem como sua especificidade; já quando se aproximam do que parece ser seu objeto, são obrigados a abandonar o método científico. Em suma, ora é psicologia não-científica, ora é ciência nãopsicológica (cf. Figueiredo, 2003).

O presente ensaio tem como objetivo mostrar uma possibilidade de superação dessas duas ameaças da psicologia científica partindo de algumas das propostas de Edward Chace Tolman (1886-1959). Inicialmente argumentamos que a adoção de uma concepção molar de comportamento afasta a ameaça do reducionismo fisiológico, na medida em que se pauta na defesa da existência de um campo comportamental irredutível à fisiologia. Essa autonomia do campo comportamental parte da existência de propriedades emergentes do comportamento, ou seja, propriedades que só podem ser encontradas no comportamento tomado como um todo, como um fenômeno molar. De acordo com Tolman (1966a [1922], 1966e [1926]), essas propriedades são propósito e cognição. Veremos que, em um primeiro momento, Tolman fundamenta essa argumentação em uma epistemologia realista, o que acaba fragilizando sua proposta.

1 Muitas vezes o mentalismo científico recai na primeira ameaça. Isso porque nesse tipo de proposta é comum a tentativa de evitar as dificuldades metodológicas por meio da tradução dos fenômenos psicológicos em termos de funcionamento cerebral. 
A saída será, então, abandonar o realismo e admitir a mediação no processo de conhecimento. Nesse ponto, surge a noção de expectativa como um conceito mediacional uma variável interveniente - que, interpretado a partir da analogia com os mapas, parece superar as limitações do realismo, sem recair no mentalismo.

\section{Comportamento gomo fenômeno molar}

Tolman pertence a uma geração de behavioristas que se dedicaram a corrigir os exageros das propostas watsonianas (cf. Smith, 1986). Esses exageros estão ligados em grande parte à filiação de Watson (1924 [1919]) ao monismo materialista (cf. Heidbreder, 1975), o que tornou sua proposta impotente frente à ameaça do reducionismo fisiológico. Ao assumir uma ontologia materialista, o behaviorismo watsoniano se viu obrigado, em algum momento, a traduzir os fenômenos comportamentais em termos físico-químicos. Com isso, a fisiologia ganha importância no estudo do comportamento, ao mesmo tempo em que a psicologia perde (cf. Tolman, 1966a [1922]).

Além disso, o monismo materialista conduziu Watson (1924 [1919]) a defender a eliminação do vocabulário mental pela psicologia científica: termos que não suportassem uma tradução fisicalista deveriam ser eliminados do vocabulário científico, com a justificativa de serem palavras vazias de significado e ligadas à tradição religiosa ou metafísica. Com isso, resta a uma psicologia científica criar um novo vocabulário para tratar dos conceitos até então considerados mentais; um vocabulário isento de conotações mentalistas, no caso, um vocabulário fisicalista. É nesse ponto que se situa grande parte das críticas tolmanianas a Watson.

Em diferentes momentos, Tolman (1966a [1922], 1967 [1932]) denuncia uma incoerência na proposta watsoniana. Em primeiro lugar, esse compromisso materialista de Watson fará com que estímulo e resposta - conceitos fundamentais para suas formulações - sejam definidos em termos puramente físico-fisiológicos: "usamos o termo ‘estímulo' em psicologia como ele é usado na fisiologia [...]. De maneira similar, empregamos na psicologia o termo fisiológico 'resposta'" (Watson, 1924 [1919], p. 10-1). Além disso, em dois dos mais importantes livros de Watson, Psychology from the standpoint of a behaviorist e Behaviorism, cerca de um terço das páginas é dedicado à descrição minuciosa das estruturas fisiológicas envolvidas no comportamento.

Mas, ao mesmo tempo em que a fisiologia invade sua proposta de psicologia, Watson continua negando explicitamente a participação da fisiologia na explicação do comportamento: 
A fisiologia não tem nada a nos dizer sobre o caráter e a personalidade de diferentes indivíduos, nem de sua estabilidade ou falta de controle emocional, nem com que extensão sua vida é dependente de sua educação. A fisiologia não nos diz nada sobre a capacidade do homem de formar e reter hábitos, nem da complexidade da organização dos hábitos dos homens (Watson, 1924 [1919], p. 20-1).

Até mesmo no caso das emoções, em que tradicionalmente se faz referência a mecanismos fisiológicos, Watson parece irredutível em relação à autonomia do behaviorismo: "no estudo das funções psicológicas - por exemplo, as emoções - não nos ajuda muito tentar esboçar quais processos neurais e químicos ocorrem no cérebro" (Watson, 1924 [1919], p. 20); ou ainda, "é perfeitamente possível para um estudante do comportamento, inteiramente ignorante acerca do sistema nervoso simpático e das glândulas e musculatura lisa, ou mesmo do sistema nervoso central como um todo, escrever um estudo completamente compreensivo e acurado das emoções" (p. 215).

Além disso, Watson não admite que o reducionismo fisiológico seja uma ameaça real para suas propostas:

Alguns psicólogos têm a noção de que o behaviorista está interessado apenas no registro momentâneo de respostas musculares. Nada pode estar mais distante da verdade. Deixe-me novamente enfatizar que o behaviorista está primariamente interessado no comportamento do homem como um todo. [...] Em outras palavras, o behaviorista está interessado na resposta à questão do senso comum 'o que ele está fazendo e por que ele está fazendo isso?' Certamente, com esse enunciado geral ninguém pode distorcer a plataforma behaviorista a ponto de alegar que o behaviorista é apenas um fisiologista dos músculos (Watson, $195^{8}$ [1924], p. 15).

Como se vê, embora defenda o comportamento como objeto de estudo autônomo, Watson acaba, em algum momento, reduzindo-o à fisiologia. Tolman (1967 [1932] entende que essa incongruência tem em sua raiz duas maneiras de considerar o comportamento, que não foram distinguidas por Watson: o comportamento como fenômeno molecular e como fenômeno molar.

Em uma visão molecular, o comportamento é entendido a partir do modelo do arco reflexo. Nessa interpretação, comportamento é um "processo que se inicia com uma excitação na superfície sensorial de um animal, é conduzido por fibras nervosas até os centros nervosos, de onde volta por nervos eferentes, e termina em uma contração muscular" (Koffka, 1935, p. 25-6). Abstraindo os componentes fisiológicos chegamos a uma definição de comportamento como uma sequência estímulo-resposta. 
No entanto, se quisermos realmente descrever todo o processo, temos que invadir o organismo e seguir a condução nervosa - presumivelmente linear -, que liga a periferia sensorial à atividade motora. Nesse ponto, o estudo do comportamento já não pode negar sua dependência da fisiologia.

Nesse sentido, o comportamento entendido como um fenômeno molecular é, em última instância, de natureza fisiológica - visto que não há diferença substancial entre as atividades internas do organismo, como circulação, respiração, digestão, e o comportamento, propriamente dito. Uma outra consequência da concepção molecular de comportamento é que ela é eminentemente reducionista. As várias ligações estímulo-resposta (mediadas por elos fisiológicos) são os fenômenos comportamentais primários e, sendo assim, tudo que observamos acerca do comportamento deve ser passível de explicação em termos de um conjunto mais ou menos complexo de séries causais de estímulos e respostas.

Para Tolman (1967 [1932]), boa parte do behaviorismo de sua época acabou filiando-se a essa concepção molecular de comportamento, o que limitou, sobremaneira, o alcance de tais propostas. Nesse sentido, o behaviorismo de Tolman consiste em uma ruptura com essa tradição. Trata-se, pois, de entender o comportamento como um fenômeno molar.

Se o comportamento como fenômeno molecular identifica-se com a visão "técnica" da maioria dos behavioristas, o comportamento como fenômeno molar coincide com a visão cotidiana que o leigo tem das ações humanas (cf. Koffka, 1935). Lembremos que, embora não a tenha levado a cabo, o próprio Watson já tinha anunciado essa pretensão: "Em outras palavras, o behaviorista está interessado na resposta à questão do senso comum 'o que ele está fazendo e por que ele está fazendo isso?'” (Watson, $195^{8}$ [1924], p. 15).

Desse modo, de um ponto de vista molar, os fatos comportamentais devem ser descritos tais como nos aparecem. É preciso, portanto, voltar-se para o comportamento como um todo, entendendo com isso que a realidade é encontrada no todo e não em suas partes. Em outras palavras, o comportamento apresenta uma realidade que só pode ser apreendida se evitarmos o ideal de análise por decomposição, típico da ciência moderna. ${ }^{2}$

Essa apreensão da natureza do comportamento como um todo inaugura, em uma perspectiva molar, um novo campo de significado, um campo comportamental, que se

2 Nesse ponto já é possível perceber que a proposta tolmaniana não está inteiramente adequada ao modelo de ciência moderna, sem com isso retornar ao subjetivismo mentalista. Além disso, é evidente a aproximação entre as propostas de Tolman e da Gestaltpsychologie. O próprio Koffka (1935), em sua obra seminal, menciona a distinção tolmaniana entre comportamento molar e molecular como um dos pontos de partida de seu projeto de psicologia científica. 
torna irredutível à fisiologia. Dessa maneira, o risco de um reducionismo fisiológico é afastado. Uma explicação do comportamento deve, portanto, recorrer às propriedades emergentes do comportamento e não a eventuais partes físico-fisiológicas. $\mathrm{O}$ desafio de uma psicologia científica não é traduzir o comportamento em termos físico-químicos, mas descrever as propriedades emergentes do comportamento.

\section{As PROPRIEDADES EMERGENTES DO GOMPORTAMENTO}

É justamente quando Tolman (1966c [1925], 1966d [1925], 1966e [1926]) define as propriedades emergentes do comportamento que surge a maior parte das acusações à sua proposta. Segundo o autor, essas propriedades são, basicamente, propósito e cognição. Ora, sem acompanhar a argumentação subjacente a essa conclusão é possível incorrer no equívoco de classificar Tolman como um mentalista: pois, aparentemente, ele está incluindo em seu sistema de comportamento conceitos de natureza "duvidosa" (mentalista). No entanto, essa interpretação não parece se sustentar. Na verdade, Tolman opera, aqui, uma diferença que será fundamental para as discussões contemporâneas no behaviorismo, trata-se da distinção entre mental e mentalista .

Ao corrigir Watson (1924 [1919]), Tolman (1966a [1922], 1966b [1923], 1966c [1925], 1966d [1925]) está nos dizendo que é possível falar de conceitos mentais no interior de um sistema comportamental, sem incorrer no mentalismo. E o que é mais importante, evitando tanto o eliminativismo quanto o reducionismo, típicos das propostas de orientação watsonianas. Mais ainda, Tolman (1966c [1925], 1966d [1925]) está nos dizendo que é possível ver a mente no comportamento. Nesse sentido, o mentalismo consiste em recusar-se a ver a mente no comportamento e, consequentemente, tentar vê-la a partir do comportamento. Em outras palavras, para o behaviorismo tolmaniano, a mente não é inferida a partir do comportamento, mas é observada no próprio comportamento. Com isso, o comportamento deixa de ser considerado como mero "sintoma" do funcionamento mental, para converter-se em um objeto de estudo legítimo de uma psicologia científica. Por caminhos razoavelmente distintos, essa mesma conclusão será alcançada por Skinner (1991 [1938]) anos depois. ${ }^{3}$

Voltando às propriedades do comportamento, Tolman (1966c [1925], 1966d [1925], 1966e [1926]) propõe uma definição behaviorista de propósito, que não envolve a introspecção, mas apenas a observação do comportamento de um organismo.

3 Nas palavras de Skinner: “Uma definição de termos em uma ciência do comportamento dada em seu próprio nível oferece a tremenda vantagem de manter o investigador consciente do que ele sabe e do que ele não sabe. O uso de termos com referências neurais, quando as observações nas quais eles são baseados são comportamentais, é um descaminho" (1991, p. 426). 
Dessa forma, propósito é entendido como a persistência em relação a um fim ou a uma meta, o que é observado nas ações de um organismo. Em outras palavras, o comportamento tem um sentido, uma direção, que podem ser inteiramente observados. Além disso, seguindo essa direcionalidade, o comportamento modifica-se em função do ambiente, de modo que o comportamento não é uma sucessão "cega" de movimentos, mas um todo coordenado pelo ambiente. Isso quer dizer que em tentativas sucessivas, não há uma mera repetição de movimentos, mas um reconhecimento do ambiente. Dessa maneira, o comportamento "exibe não apenas propósitos, mas também postulações cognitivas sobre a natureza do ambiente na mediação e no suporte de propósitos" (Tolman, 1966e [1926], p. 51).

\section{UM PROBLEMA NA PROPOSTA MOLAR DE TOLMAN}

A dificuldade que surge nessa proposta diz respeito à natureza das propriedades do comportamento. Em outras palavras, será que o comportamento é realmente propositivo e cognitivo? Ou será que é o observador que interpreta o comportamento dessa maneira? Isso parece ameaçar o próprio projeto de estudo molar do comportamento. Se admitirmos que o comportamento não é "realmente" propositivo e cognitivo, não estaríamos falsificando a visão molar que se baseia nessa realidade?

À primeira vista só resta a Tolman admitir a realidade das propriedades do comportamento. Encontramos essa afirmação nos seus primeiros textos (Tolman, 1966a [1922], 1966c [1925], 1966d [1925], 1966e [1926]), nos quais admite que as propriedades do comportamento molar estão no comportamento. Segundo essa proposta, propósito e cognição são diretamente observáveis e, portanto, não há qualquer inferência no processo de conhecimento dessas propriedades (Tolman, 1966c [1925], 1966e [1926]). Em outras palavras, propósito e cognição estão no comportamento e não atrás dele. Nesse sentido, tentar inferir propósito do comportamento é filiar-se ao mentalismo. Nas palavras de Tolman:

a diferença fundamental entre ele [Mc Dougall] e nós surge do fato de que, sendo um "mentalista", ele meramente infere propósito desses aspectos do comportamento; enquanto que nós, sendo behavioristas, identificamos propósito com esses aspectos (1966c [1925], p. 33).

Com isso, Tolman (1966c [1925], 1966d [1925], 1966e [1926]) parece resolver o problema. Ficamos no campo do comportamento como fenômeno molar com a justificativa de que o comportamento é realmente propositivo e cognitivo. Quando olhamos 
para o comportamento vemos propósito e cognição. Nesse ponto, Tolman filia-se a um tipo de realismo. ${ }^{4}$

O que subjaz a essa posição é a crença de que o processo de conhecimento é direto. Ou seja, quando um organismo estabelece contato com o ambiente, ele o faz de maneira passiva, pois tudo está no ambiente, sendo, por isso, diretamente apresentado ao observador (cf. Passmore, 1986). No caso do comportamento, sua realidade apresenta-se integralmente para o observador. Um dos problemas desse posicionamento realista é que ele pode acabar levando ao dogmatismo. Ora, se as coisas (assim como a mente) se apresentam integralmente para o observador há apenas uma possibilidade de conhecimento: aquele que descreve o que realmente está apresentado.

Smith (1986) analisa bem a crescente dificuldade enfrentada por Tolman para sustentar essa epistemologia realista. $\mathrm{O}$ argumento realista de que a mente é vista diretamente no comportamento foi questionado até mesmo por outros behavioristas da época, obrigando Tolman, já em 1928, a admitir que essas propriedades eram inferidas do comportamento e não diretamente percebidas. Essa hesitação em relação à epistemologia realista refletir-se-á em uma mudança em relação à natureza do propósito e da cognição, que ocorre a partir da segunda metade dos anos 1920: aos poucos vão deixando de ser consideradas propriedades do comportamento para tornarem-se determinantes do comportamento (cf. Smith, 1986).

De fato, a visão passiva de conhecimento não se ajustava com alguns dados experimentais (cf. Smith, 1986). No início da década de 1930, Kreschevsky - na época aluno de Tolman - conduziu uma série de experimentos em que ratos eram reforçados aleatoriamente em labirintos em “T”. Em outras palavras, sem um padrão específico o alimento era disposto ora em um braço do labirinto, ora em outro. Seguindo uma interpretação passiva de conhecimento, deveríamos esperar que o comportamento do rato fosse inteiramente sensível a essas mudanças e que não houvesse, portanto, qualquer padrão no comportamento desses animais. Os resultados foram exatamente opostos. Nessa situação, os ratos apresentavam padrões bastante estáveis e, o que é mais surpreendente, variáveis de sujeito para sujeito. Ou seja, enquanto um rato seguia um padrão de duas tentativas no braço esquerdo para uma tentativa no direito, por exemplo, outro seguia um padrão de cinco tentativas na direita para duas na esquerda.

Isso sugeria que os ratos não eram apenas expectadores das condições experimentais, mas participavam ativamente da construção do conhecimento desse ambiente. Para explicar esse papel ativo, Krechevsky empregou o conceito de hipótese (cf. Smith,

4 Na verdade, trata-se de uma filiação ao neo-realismo de R. B. Perry e E. B. Holt, professores de Tolman em Harvard (cf. Smith, 1986). Segundo esses autores, a mente se revela inteira e diretamente no comportamento (cf. Tolman, 1966c). Assim, quando falamos da mente de uma pessoa, não há qualquer processo de inferência envolvido, mas apenas a observação de seu comportamento (cf. Mora, 2001; Passmore, 1986). 
1986). Em um ambiente específico, os ratos construíam hipóteses sobre o "funcionamento" desse ambiente e perseguiam essas hipóteses - o que era revelado pelos padrões persistentes observados. O conhecimento era, portanto, mediado por hipóteses, o que contrariava a concepção realista de conhecimento direto.

Com isso, ganha força uma concepção menos dogmática acerca da explicação do comportamento: agora não é mais possível considerar o mentalismo como um tipo de “miopia”, que se afasta da única visão correta acerca do comportamento, mas é preciso entendê-lo como uma proposta, dentre as possíveis interpretações do comportamento. Nesse ponto, entretanto, o projeto tolmaniano encontra nova dificuldade: se não há mais um critério de realidade, e se as hipóteses que explicam as ações observadas não são diretamente observadas, mas sim inferidas, Tolman não estaria novamente recaindo no mentalismo?

\section{O Lugar dAS EXPEGTativas No SISTEMA DE TOLMAN}

Antes de tentar responder a questão colocada na seção anterior, convém situar a noção de hipóteses no sistema de comportamento proposto por Tolman (1967 [1932]). Para incluir a mediação no processo de conhecimento, Tolman empregará não o conceito de hipótese, mas o de expectativa. Entretanto, o sentido de expectativa, bem como os problemas subjacentes a seu emprego, são praticamente os mesmos do conceito de hipótese.

As expectativas são entendidas por Tolman (1967 [1932]) como um tipo de variável interveniente que, como o nome sugere, são determinantes do comportamento situados entre as variáveis independentes (estímulo atual, hereditariedade, treino prévio e estados fisiológicos) e as ações observáveis. As variáveis intervenientes constituem um tipo de estado comportamental (um conjunto de capacidades, tendências e prontidões para agir de uma dada maneira), cuja origem é explicada basicamente pela dotação genética e pela história de treino de um organismo. Nesse sentido, um comportamento atual é sempre uma função da inter-relação entre variáveis independentes e intervenientes.

Portanto, dada uma configuração de variáveis independentes temos um conjunto de variáveis intervenientes que tornam mais ou menos provável um tipo de ação. Ora, uma vez que as variáveis intervenientes não são diretamente observáveis, elas devem ser inferidas a partir da relação entre variáveis independentes e ações observáveis (Tolman, 1967 [1932]). A questão mais geral diz respeito, então, à natureza das variáveis intervenientes. Se admitirmos que os referentes dessas variáveis estão em um mundo mental, recaímos no mentalismo. Tentando escapar desse embaraço pode- 
mos situar os referentes no cérebro, mas então nos vemos às voltas com o reducionismo fisiológico. Haveria uma alternativa?

\section{O PRAGMATISMO GOMO SOLUÇÃO}

As questões suscitadas pelo abandono de uma epistemologia realista serão enfrentadas por Tolman (1967 [1932]) por meio de uma análise do estatuto das teorias científicas. A questão que se coloca nesse contexto é qual a função das teorias científicas. Em uma perspectiva realista, como a inicialmente adotada por Tolman (1966a [1922], 1966c [1925], 1966d [1925], 1966e [1926]), teorias são descrições fiéis da realidade. Dessa forma, o tipo de relação entre teoria e fato em uma epistemologia realista é o de espelhamento. E, portanto, a verdade de uma teoria é aferida por sua correspondência com os fatos (cf. Nagel, 1961).

Já em uma epistemologia não-realista, a teoria não tem mais a pretensão de espelhar, ou mesmo descrever, a realidade. No caso de Tolman (1967 [1932]), a função da teoria passa a ser operar sobre a realidade de maneira efetiva; o que, no contexto de sua proposta de ciência, significa promover previsão e controle do comportamento. Assim, nessa nova epistemologia, a verdade de uma teoria passa a ser aferida por sua utilidade, por sua capacidade de conduzir o cientista a alcançar seus objetivos.

Nesse ponto não é difícil perceber a aproximação de Tolman (1967 [1932]) com o instrumentalismo científico (Nagel, 1961). Trata-se, pois, de assumir o caráter estritamente instrumental das teorias, bem como o critério pragmatista de verdade como efetividade. Na esteira de sua filiação com esse tipo de pragmatismo, Tolman (1967 [1932]) defenderá que a relação entre teoria científica e fato é apreendida por meio da analogia com os mapas.

Analisemos essa analogia com um exemplo. Imaginemos que precisamos ensinar uma pessoa a chegar à universidade, saindo do centro da cidade. Para tanto podemos fazer um mapa, que mostra pontos de referência, distâncias, ou apenas direções. Se o mapa alcançar seu objetivo - levar a pessoa até à universidade -, não preciso me preocupar se ele estava em escala, se ignorou uma série de outros aspectos do ambiente, e assim por diante. Importa que o mapa cumpriu sua função: levar a pessoa de um lugar a outro. Para Tolman (1967 [1932]), o mesmo raciocínio deve ser feito em relação às teorias científicas. Quando o comportamento é descrito por meio dos conceitos de expectativa, propósito, cognição, etc. deveríamos nos preocupar menos com a correspondência desses conceitos com uma suposta "realidade" do comportamento, e mais com a possibilidade de alcançarmos previsão e controle. 
Seguindo essa analogia entre mapas e teorias, Tolman (1967 [1932]) defenderá que uma das características da ciência é construir mapas - teorias úteis -, o que quer dizer que toda ciência trabalha distorcendo a realidade a fim de alcançar seus objetivos:

Como cientistas começamos com a experiência imediata, real e rica, mas, buscando previsão e controle, terminamos com demandas, prontidões-meio-fim, expectativas-sinal-gestalt e todo o resto. Mas podemos supor que essa distorção e emasculação das realidades da experiência imediata não é mais violenta e repulsiva do que aquela proposta pelo físico ou pelo químico. Esses pensadores também começam com realidades concretas e ricas - cadeiras, mesas, relógios, tintas. Mas, interessados em previsão e controle, eles não acabam com elétrons e prótons, ou ondas? (Tolman, 1967 [1932], p. 424).

Se transpusermos essa discussão para as questões anteriormente formuladas, o conceito de mapa parece indicar uma solução para o impasse. Ao defender que o conhecimento era mediado e que, portanto, as variáveis intervenientes participavam da explicação do comportamento, a proposta tolmaniana parecia estar novamente ameaçada pelo mentalismo e pelo reducionismo fisiológico. No entanto, assumindo uma concepção pragmatista de teoria científica - por meio da analogia com os mapas -, Tolman (1967 [1932]) parece afastar-se dessas ameaças.

Assim, em uma perspectiva pragmatista, as variáveis intervenientes podem ser entendidas como mapas ou instrumentos criados pelos cientistas a fim de aprimorar sua explicação do comportamento e, consequentemente, alcançar previsão e controle. Nesse sentido, o fato de as variáveis intervenientes não serem observáveis, mas inferidas, não compromete Tolman (1967 [1932]) nem com o mentalismo, nem como o reducionismo fisiológico. Essas ameaças só aparecem no contexto de uma epistemologia realista, que defende uma relação de espelhamento entre conceito e fato: mentalismo quando o conceito espelha (ou tem como referente) uma realidade mental; e reducionismo fisiológico quando espelha uma realidade neurofisiológica.

\section{Conclusão}

Alguns aspectos da proposta de psicologia científica de E. C. Tolman parecem afastar seu projeto tanto do reducionismo fisiológico, quanto do mentalismo. A primeira investida nesse sentido pode ser encontrada na proposta de um estudo molar do comportamento. Contrapondo-se a Watson (1924 [1919], $195^{8}$ [1924]), Tolman (1966a [1922], 1966b [1923], 1966c [1925], 1966d [1925], 1966e [1926]) propõe que o com- 
portamento deve ser considerado como um todo dotado de propriedades emergentes. A identificação dessas propriedades inaugura um campo comportamental irredutível à fisiologia - pois quando nos voltamos para as eventuais partes fisiológicas do comportamento, tais propriedades desaparecem.

Essa proposta fundamenta-se inicialmente em uma epistemologia realista (Tolman, 1966a [1922]). Visto que nesse tipo de epistemologia a função das teorias é espelhar os fatos, Tolman afirmará que as propriedades emergentes estão realmente no comportamento, ou seja, que pertencem à sua natureza. Dessa maneira, Tolman barra tanto o reducionismo fisiológico - que ao se voltar para partes fisiológicas do comportamento, acaba destruindo as propriedades definidoras do comportamento -, quanto o mentalismo - que simplesmente não é capaz de enxergar tais propriedades.

Uma das principais dificuldades encontradas por Tolman para levar essa proposta adiante aparecerá com alguns dados experimentais: no início da década de 193o, estudos realizados por um de seus alunos apontaram que o comportamento de ratos seguia padrões específicos - mesmo não havendo correspondência entre esses padrões e as exigências ambientais (cf. Smith, 1986). Isso sugeria que os ratos tinham uma participação ativa no processo de conhecimento de seu ambiente. Na tentativa de explicar esse fato Tolman (1967 [1932]) admitirá que o comportamento não é diretamente determinado pelo ambiente, mas por variáveis que se interpõem entre o organismo e o ambiente - as variáveis intervenientes.

Nesse ponto, a proposta tolmaniana encontra nova dificuldade. Uma vez que essas variáveis não são diretamente observadas (na verdade são inferidas do comportamento observado), parece que as duas ameaças ganham espaço novamente: se Tolman admitir que as variáveis inferidas têm como referentes processos mentais subjacentes ao comportamento, sua proposta filia-se ao mentalismo; se tentar evitar essa filiação, admitindo que elas têm referentes neurofisiológicos, está diante do reducionismo fisiológico.

A saída encontrada por Tolman (1967 [1932]) será abandonar definitivamente uma epistemologia realista e assumir uma epistemologia instrumentalista. Essa adesão ao instrumentalismo aparece na analogia que Tolman propõe entre teorias científicas e mapas. Tal como os mapas, as teorias não são espelhos da realidade, mas um conjunto de instrumentos conceituais que guiam o cientista até seus objetivos.

Nesse sentido, em uma epistemologia instrumentalista não cabe perguntar pelos referentes das variáveis intervenientes - o que seria fundamental em uma epistemologia realista. A função do conceito não é espelhar uma realidade, mas apenas conduzir o cientista a seus objetivos - que, no caso da psicologia tolmaniana, é a previsão e controle do comportamento. Portanto, a natureza das variáveis intervenientes não é nem mentalista, nem neurofisiológica; é simplesmente teórica. 


\author{
Carlos Eduardo Lopes \\ Professor Doutor do Departamento de Psicologia, \\ Centro de Ciências Humanas, Letras e Artes, \\ Universidade Estadual de Maringá, Brasil. \\ caedlopes@gmail.com
}

\begin{abstract}
Every project of scientific psychology faces at least two challenges. The first challenge appears when a proposal of psychology tries to follow the standard of modern science. In this case, it is necessary to make the psychological phenomena objective, what is done, most frequently, by means of its translation into physiological terms. In consequence, the specificity of psychology becomes threatened by physiological reductionism. The second challenge appears when a project of psychology tries to avoid the physiological reductionism by means of defending the irreducible and subjective nature of psychological phenomena. In fact, this kind of mentalism limits the application of scientific method. This essay intends to show how E. C. Tolman's project of scientific psychology could be an alternative for those challenges. In first place, we show that Tolman keeps himself away from the physiological reductionism in virtue of his molar conception of behavior. Next, we analyze some difficulties of his proposal, especially the limitations of a realistic epistemology. Finally, we present Tolman's proposal for surmounting such difficulties: the analogy of scientific theories considered as maps, which reveals his mature instrumentalist epistemology.
\end{abstract}

KEYwords • Tolman. Behavior. Maps. Physiological reductionism. Mentalism.

\title{
REFERÊNGIAS BIBLIOGRÁFIGAS
}

Araujo, S. F. Psicologia e neurociência: uma avaliação da perspectiva materialista dos fenômenos mentais. Juiz de Fora: Editora da UFJF, 2003.

Figueiredo, L. C. M. Matrizes do pensamento psicológico. Petrópolis: Vozes, 2003.

Heidbreder, E. Psicologias do século XX. São Paulo: Mestre Jou, 1975 [1933].

KoffKa, K. Principles of gestalt psychology. New York: Harcourt, Brace \& Company, 1935.

Mora, J. F. Dicionário de filosofia. São Paulo: Loyola, 2001 [1994]. t. 3.

NAGEL, E. The structure of science: problems in the logic of scientific explanation. New York: Harcourt, Brace \& World, 1961.

Passmore, J. A hundred years of philosophy. New York: Penguin Books, 1986.

Skinner, B. F. Behavior of organisms. Massachusetts: Acton, 1991 [1938].

Sмітн, L. D. Behaviorism and logical positivism: a reassessment of the alliance. Stanford: Stanford University Press, 1986.

Tolman, E. G. Behavior and psychological man: essays in motivation and learning. 4. ed. Berkeley: University of California Press, 1966.

. A new formula for behaviorism. In:

Behavior and psychological man: essays in motivation and learning. 4. ed. Berkeley: University of California Press, 1966a [1922]. Cap. 1, p. 1-8.

. A behavioristic account of the emotions. In: Behavior and psychological man:essays in motivation and learning. 4. ed. Berkeley: University of California Press, 1966b [1923]. Cap. 3, p. 23-31. 
Tolman, E. C. Behaviorism and purpose. In: . Behavior and psychological man: essays in motivation and learning. 4. ed. Berkeley: University of California Press, 1966c [1925]. Cap. 4, p. 32-7.

Purpose and cognition: the determiners of animal learning. In: Behavior and psychological man: essays in motivation and learning. 4. ed. Berkeley: University of California Press, 1966d [1925]. Cap. 5, p. 38-47.

A behaviorist theory of ideas. In: Behavior and psychological man: essays in motivation and learning. 4. ed. Berkeley: University of California Press, 1966e [1926]. Cap. 6, p. 48-62.

Purposive behavior in animals and men. 2. ed. New York: Appleton Century Croft, 1967 [1932].

Watson, J. B. Psychology from the standpoint of a behaviorist. 2. ed. Philadelphia: J. B. Lippincott, 1924 [1919]

. Behaviorism. 4. ed. Chicago: The University of Chicago Press/Phoenix Books, $195^{8}$ [1924].

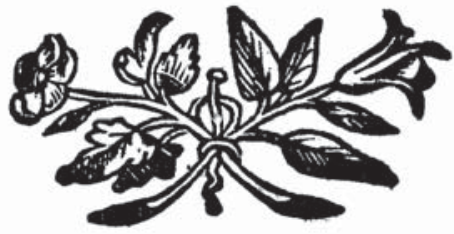

